\title{
Correction to: Dietary intake of soy and cruciferous vegetables and treatment-related symptoms in Chinese-American and non-Hispanic White breast cancer survivors
}

\author{
Sarah J. O. Nomura ${ }^{1}$ Yi-Ting Hwang ${ }^{2}$. Scarlett Lin Gomez ${ }^{3}$. Teresa T. Fung ${ }^{4,5}$. Shu-Lan Yeh ${ }^{6}$ Chiranjeev Dash ${ }^{1}$. \\ Laura Allen $^{3} \cdot$ Serena Philips ${ }^{7} \cdot$ Leena Hilakivi-Clarke $^{1} \cdot$ Yun-Ling Zheng ${ }^{1} \cdot$ Judy Huei-yu Wang $^{1,8}(\mathbb{0}$
}

Published online: 11 January 2018

(c) Springer Science+Business Media, LLC, part of Springer Nature 2018

\section{Correction to: Breast Cancer Research and Treatment https://doi.org/10.1007/s10549-017-4578-9}

In the original publication, the values provided for the isoflavone and glucosinolate intake variables were incorrectly labeled in Table 1. The correct values of $6.3 \mathrm{mg} /$ day for isoflavone intake, and $20.4 \mathrm{mg} / \mathrm{day}$ and $50.1 \mathrm{mg} /$ day for glucosinolate intake are provided in this erratum. Under the "Statistical Analysis" section, second paragraph, the values in the 11th line "...time since diagnosis $(<4,3-36$, and $\geq 36$ months)..." was mislabeled. The correct values were " $<24,24-36$, and $>36$ months".

The original article can be found online at https://doi.org/10.1007/ s10549-017-4578-9.

Judy Huei-yu Wang jw235@georgetown.edu

1 Department of Oncology, Lombardi Comprehensive Cancer Center, Georgetown University, 3800 Reservoir Rd NW, Washington, D.C. 20057, USA

2 Department of Statistics, National Taipei University, Taipei, Taiwan

3 Cancer Prevention Institute of California, Fremont, CA, USA

4 Department of Nutrition, Simmons College, Boston, MA, USA

5 Department of Nutrition, Harvard T. H. Chan School of Public Health, Boston, MA, USA

6 Department of Nutrition, Chang Shan Medical University, Taichung, Taiwan

7 Milken Institute School of Public Health, George Washington University, Washington, D.C. 20052, USA

83300 Whitehaven Street, NW, Suite 4100, Washington, D.C. 20007, USA
Table 1 Population characteristics

\begin{tabular}{|c|c|c|c|}
\hline & $\begin{array}{l}\text { Total popula- } \\
\text { tion }\end{array}$ & $\begin{array}{l}\text { Non-Hispanic } \\
\text { White }\end{array}$ & $\begin{array}{l}\text { Chinese } \\
\text { American }\end{array}$ \\
\hline Total $N(\%)$ & $365(100)$ & $173(47.4)$ & $192(52.6)$ \\
\hline $\begin{array}{l}\text { Age in years (mean, } \\
\text { SD) }\end{array}$ & $57.1(10.4)$ & $57.2(10.5)$ & $56.9(10.4)$ \\
\hline \multicolumn{4}{|c|}{ Menopausal status $(N, \%)$} \\
\hline Premenopause & $47(13.0)$ & $21(12.3)$ & $26(13.7)$ \\
\hline Induced menopause & $61(16.9)$ & $30(17.5)$ & $31(16.3)$ \\
\hline Perimenopause & $81(22.4)$ & $35(20.5)$ & $46(24.2)$ \\
\hline Postmenopause & $172(47.6)$ & $85(49.7)$ & $87(45.8)$ \\
\hline $\begin{array}{l}\mathrm{BMI}\left(\mathrm{kg} / \mathrm{m}^{2}\right)(\text { mean, } \\
\text { SD) }\end{array}$ & $24.0(4.4)$ & $25.2(5.1)$ & $22.9(3.1)$ \\
\hline \multicolumn{4}{|c|}{ Physical activity $(N, \%)^{\mathrm{a}}$} \\
\hline Active & $110(30.1)$ & $77(44.5)$ & $33(17.2)$ \\
\hline Minimally active & $136(37.3)$ & $58(33.5)$ & $78(40.6)$ \\
\hline Inactive & 119 (32.6) & $38(22.0)$ & $81(42.2)$ \\
\hline \multicolumn{4}{|l|}{ Cancer stage $(N, \%)$} \\
\hline Stage 0 & $110(30.1)$ & $64(37.0)$ & $46(24.0)$ \\
\hline Stage I & $166(45.4)$ & $77(44.5)$ & $89(46.4)$ \\
\hline Stage II & 47 (12.9) & $22(12.7)$ & $25(13.0)$ \\
\hline Stage III & $42(11.5)$ & $10(5.8)$ & $32(16.7)$ \\
\hline \multicolumn{4}{|c|}{ Endocrine therapy $(N, \%)$} \\
\hline None & $148(40.8)$ & $74(42.8)$ & $74(38.5)$ \\
\hline Tamoxifen & $126(34.5)$ & $60(34.7)$ & $66(34.4)$ \\
\hline Aromatase inhibitor & $91(24.9)$ & $39(22.5)$ & $52(27.1)$ \\
\hline \multicolumn{4}{|l|}{ Lumpectomy $(N, \%)$} \\
\hline No & $141(38.6)$ & $59(34.1)$ & $82(42.5)$ \\
\hline Yes & $224(61.4)$ & $114(65.9)$ & $110(57.3)$ \\
\hline \multicolumn{4}{|l|}{ Mastectomy $(N, \%)$} \\
\hline No & $228(62.5)$ & $114(65.9)$ & $114(59.4)$ \\
\hline Yes & $137(37.5)$ & $59(34.1)$ & $78(40.6)$ \\
\hline \multicolumn{4}{|l|}{ Chemotherapy $(N, \%)$} \\
\hline No & $257(76.3)$ & $127(80.4)$ & $130(72.6)$ \\
\hline Yes & $80(23.7)$ & $31(19.6)$ & $49(27.4)$ \\
\hline
\end{tabular}


Table 1 continued

\begin{tabular}{|c|c|c|c|}
\hline & $\begin{array}{l}\text { Total popula- } \\
\text { tion }\end{array}$ & $\begin{array}{l}\text { Non-Hispanic } \\
\text { White }\end{array}$ & $\begin{array}{l}\text { Chinese } \\
\text { American }\end{array}$ \\
\hline \multicolumn{4}{|c|}{ Radiation therapy $(N, \%)$} \\
\hline No & $198(54.2)$ & $88(50.9)$ & $110(57.3)$ \\
\hline Yes & $167(45.8)$ & $85(49.1)$ & $82(42.7)$ \\
\hline \multicolumn{4}{|c|}{ Time since diagnosis $(N, \%)$} \\
\hline$<24$ months & $108(29.6)$ & $48(27.7)$ & $60(31.3)$ \\
\hline 24-36 months & $111(30.4)$ & $49(28.3)$ & $62(32.3)$ \\
\hline$>36$ months & $146(40.0)$ & $76(43.9)$ & $70(36.5)$ \\
\hline \multicolumn{4}{|l|}{ Estrogen receptor $(N, \%)$} \\
\hline Positive & $227(62.2)$ & $109(63.0)$ & $118(61.5)$ \\
\hline Negative & 49 (13.4) & $19(11.0)$ & $30(15.6)$ \\
\hline Unknown & $89(24.4)$ & $45(26.0)$ & $44(22.9)$ \\
\hline \multicolumn{4}{|c|}{ Progesterone receptor $(N, \%)$} \\
\hline Positive & $196(53.7)$ & $91(52.6)$ & $105(54.7)$ \\
\hline Negative & $80(21.9)$ & $37(21.4)$ & $43(22.4)$ \\
\hline Unknown & $89(24.4)$ & $45(26.0)$ & $44(22.9)$ \\
\hline \multicolumn{4}{|l|}{$\operatorname{HER} 2(N, \%)$} \\
\hline Positive & $44(12.1)$ & $18(10.4)$ & $26(13.5)$ \\
\hline Negative & $167(45.8)$ & $74(42.8)$ & $93(48.4)$ \\
\hline Unknown & $154(42.2)$ & $81(46.8)$ & $73(38.0)$ \\
\hline \multicolumn{4}{|l|}{ Soy products $(N, \%)^{\mathrm{b}}$} \\
\hline No intake & $104(28.5)$ & $73(42.2)$ & $31(16.1)$ \\
\hline$>0-<24.0 \mathrm{~g} /$ day & $131(35.9)$ & $65(37.6)$ & $66(34.4)$ \\
\hline$\geq 24.0 \mathrm{~g} /$ day & $130(35.6)$ & $35(20.2)$ & $95(49.5)$ \\
\hline \multicolumn{4}{|l|}{ Isoflavones $(N, \%)^{\mathrm{b}}$} \\
\hline No intake & $104(28.5)$ & $73(42.2)$ & $31(16.1)$ \\
\hline$>0-<6.3 \mathrm{mg} /$ day & $129(35.3)$ & $62(35.8)$ & $67(34.9)$ \\
\hline$\geq 6.3 \mathrm{mg} /$ day & $132(36.2)$ & $38(22.0)$ & $94(49.0)$ \\
\hline \multicolumn{4}{|c|}{ Cruciferous vegetables $(N, \%)^{\mathrm{b}}$} \\
\hline$<33.0 \mathrm{~g} /$ day & $121(33.2)$ & $77(44.5)$ & $44(22.9)$ \\
\hline$\geq 33.0-<70.8$ g/day & $120(32.9)$ & $58(33.5)$ & $62(32.3)$ \\
\hline$\geq 70.8 \mathrm{~g} /$ day & $124(33.9)$ & $38(22.0)$ & $86(44.8)$ \\
\hline \multicolumn{4}{|l|}{ Glucosinolates $(N, \%)^{\mathrm{b}}$} \\
\hline$\leq 20.4 \mathrm{mg} /$ day & $121(33.2)$ & $70(40.5)$ & $51(26.6)$ \\
\hline $\begin{array}{l}>20.4-<50.1 \mathrm{mg} / \\
\text { day }\end{array}$ & $120(32.9)$ & $55(31.8)$ & $65(33.9)$ \\
\hline$\geq 50.1 \mathrm{mg} /$ day & $124(33.9)$ & 48 (27.7) & 76 (39.6) \\
\hline
\end{tabular}

${ }^{a}$ Active: vigorous physical activity $\geq 3$ days/week $+\geq 1500$ METminutes/week or $\geq 3000$ MET-minutes/week engaged in any intensity levels of physical activity; minimally active: $<$ Active cut-points and $\geq 600$ MET-minutes/week; inactive: < 600 MET-minutes/week

${ }^{\mathrm{b}}$ Tertiles
The authors wish to clarify the description of a prior study by Dorjgochoo et al. (2011) in the discussion section: "an observational study among Chinese survivors found that higher soy food intake (45-47 $\mathrm{mg} /$ day) increased hot flashes at 6 or 36 months post-breast cancer diagnosis [16]". The phrase "higher soy food intake (45-47 mg/day)" should have been "higher soy isoflavone intake (> $62.86 \mathrm{mg} /$ day)". The Dorjgochoo et al. study was referring to soy isoflavone intake specifically and $45-47 \mathrm{mg}$ /day actually refers to the average daily intake of soy isoflavone. Additionally, the authors stated that the Dorjgochoo et al. study "only assessed hot flashes as menopausal symptom outcome". It should be clarified that the Dorjgochoo et al. study assessed multiple menopausal symptoms but the current study assessed more.

The clarifications mentioned in this erratum do not influence or modify the results and conclusions reported in the original article. 